\title{
Acogimento familiar: construcción histórica de su institucionalidad en la pampa argentina
}

Carolina Farias Carracedo*

fariascarracedo@gmail.com

Andrea Piñeda**

mandreapineda@hotmail.com

Hugo Klappenbach***

hklappen@gmail.com

\section{Resumen}

En este trabajo analizamos el recorrido histórico del Programa de Acogimiento Familiar en la Provincia de La Pampa (Argentina), desde sus inicios en 1967 hasta la actualidad. Tiene como objetivo aportar al conocimiento sobre el funcionamiento de estos programas en Argentina así como analizar el impacto real, sobre las políticas públicas de infancia, de la Convención Internacional de los Derechos del Niño y su consecuente Ley Nacional de Protección Integral. Metodológicamente, se trabajó desde un enfoque ex post facto retrospectivo con utilización de fuentes primarias y secundarias. A partir de los resultados obtenidos, hemos señalado tres etapas diferenciadas en el programa. La primera, desde el año 1967 al 2000, cuya denominación fue "Hogares Sustitutos". Luego, en el año 2000 pasa a llamarse "Familias de Contención". La tercera etapa se inicia en el año 2013, cuando la provincia adhirió a la Ley Nacional de Protección Integral de los Derechos de las Niñas, Niños y Adolescentes, que había sido sancionada en el año 2005. Se concluye que, a pesar del esfuerzo que la provincia viene realizando por adecuar sus prácticas a la legislación, las políticas públicas y las prácticas institucionales relativas al acogimiento familiar presentar serias dificultades que aún no han sido saldados.

\section{Palabras claves}

Acogimiento Familiar; Derecho de las Niñas, Niños y Adolescentes; Historia

\section{Foster care: historical construction of its institutionality in La Pampa, Argentina}

\begin{abstract}
This paper provides with a historical analysis about the Program of Foster Care in La Pampa (Argentina), from its starting point in 1967 to the present time. We aim at making a contribution to the knowledge of this program in Argentina, as there are incomplete surveys which do not include La Pampa. On the other hand, historical data is offered to analyze the incidence that the International Convention of the Children Rights and the National Law of Integral Protection of Children had on local public policies. The historical reconstruction was made upon primary and secondary sources. Results allowed us to make a periodization of the program, including three different stages. First, from 1967 to 2000, when the "Foster Homes" Program took place. Secondly, from 2000 to 2013, when there was the "Foster Family" Program. In 2013, it begun the third period, when the Government of La Pampa ratified the national law of Integral Protection of Children enacted in 2005. Data provided, sustain the conclusion that, in spite of La Pampa Government's efforts to adecuate foster care policies and institutional practices to the current legislation, there are deep problems to solve yet.
\end{abstract}

\section{Keywords}

Foster Care; Children Rights; History 
El futuro de los niños es siempre hoy. Mañana será tarde.

Gabriela Mistral

\section{Introducción}

En el presente trabajo analizamos el recorrido histórico del Programa de Acogimiento Familiar (AF) en la Provincia de La Pampa (Argentina), desde sus inicios en 1967 hasta la actualidad. Con el mismo nos proponemos contribuir en dos sentidos. En primer lugar, proponemos recuperar datos empíricos sobre la historia de este programa, a los fines de complementar la escasa información disponible sobre la región, que en algunos casos es confusa, parcial o errónea. De esa manera, buscamos ampliar el conocimiento sobre el funcionamiento de estos programas en Argentina, y mostrar las peculiaridades del contexto local.

Por otra parte, desde esta historia local, quisiéramos contribuir a visibilizar una problemática poco estudiada, la de los niños y niñas carentes de cuidados parentales. Dicho problema se materializa en un colectivo cuyos derechos han sido proclamados por la Convención Internacional de los Derechos del Niño y deben ser garantizados, según la Ley Nacional de Protección Integral de los Derechos de las Niñas, Niños y Adolescentes (ley 26.061 del año 2005). No obstante, las políticas públicas y las prácticas institucionales relativas al acogimiento familiar suelen ser motivo de debates públicos que aún no han sido saldados.

Definimos la institución "acogimiento familiar" como aquella práctica donde un niño que, por diversos motivos no puede permanecer con sus progenitores, es aceptado por otra familia (familia extensa o sin vínculos de parentesco) para cuidarlo e integrarlo como un miembro más de esa familia. Se trata de un proceso que implica una medida transitoria, hasta que se encuentre una solución definitiva para cada niño en particular (Farias-Carracedo; Piñeda, 2017).

Aquí nos referiremos solo a los casos de AF formal, es decir, aquellas prácticas que se formalizan porque actúan órganos estatales judiciales y/o administrativos involucrados en todo el proceso. Así, no consideramos aquí aquellos casos de AF informal, donde una familia se responsabiliza de un niño que no ha nacido en ella sin que medie ningún tipo de intervención estatal.

Aun cuando no hay una gran producción teórica referida al AF, los aportes a nivel Latinoamericano son enriquecedores para aquellos que trabajan en la temática.

La Red Latinoamericana de Acogimiento Familiar (RELAF) ha realizado numerosos aportes a la comprensión del funcionamiento en esta región. En especial, señalamos los documentos dirigidos a quienes trabajan en estos programas: relevamientos de datos respecto a niños sin cuidados parentales, así como aquellos que analizan la situación de vulneración de derechos en la infancia (RELAF,2013, s/f; RELAF\&UNICEF, 2010a, 2010b).

A nivel nacional, gran parte de las producciones teóricas pertenecen a Matilde Luna y colaboradores, pero, responden a la realidad de Buenos Aires, quedando ausente el análisis de otras regiones geográficas (Luna, 1994, 1998, 2001, 2005, 2006, 2009; RELAF, 2011). Asimismo, resaltamos las consideraciones de Marisa Herrera, proveniente del campo del Derecho (2008).

De la misma manera, en nuestro país, desde el año 2013, se inició el proceso de formación de la Red Federal de Acogimiento Familiar, la cual surgió desde el interés de los profesionales que estaban trabajando en estos programas de conocer las realidades del resto de las provincias (Red Federal de Acogimiento Familiar, 
Carolina Farias Carracedo - Andrea Piñeda • Hugo Klappenbach

2016). Este organismo ha publicado recientemente, en enero de 2018, el primer informe sobre la situación actual del acogimiento en Argentina, que data de un relevamiento, llevado a cabo durante el transcurso del año 2016, acerca de los programas de acogimiento familiar. Como el mismo informe lo indica, presenta de manera descriptiva los diversos programas recabados en las diferentes regiones con información suministrada por referentes de cada programa comunal, municipal o provincial (Red Federal de Acogimiento Familiar, 2018). Posiblemente, la modalidad de búsqueda de información ha llevado a que algunos datos presentados, para el caso de la provincia de La Pampa, no sean correctos. Nos proponemos aquí salvar aquella información sin desconocer el enorme esfuerzo y el valor del emprendimiento en la tarea que la Red Federal se encuentra realizando.

Respecto a las investigaciones en las provincias del país, también son escasas. Entre ellas, los aportes de Daniel Ocampo, licenciado en Servicio Social, en la provincia de Neuquén hace contribuciones sobre el funcionamiento del programa (Ocampo, 2012, s/f). Para el caso de la provincia de Mendoza, se encuentran algunos análisis, desde la psicología, acerca del programa como es el caso de Alma Férnandez-Hasan (2008, 2011), Julieta Fernández (2010) y Mónica Valgañón (2014).

Ahora bien, en ninguno de estos antecedentes aludidos, se encuentran referencias relevantes acerca de la historia de esta modalidad de albergue. Matilde Luna (2006) en su libro "Acogimiento Familiar, Respuesta social y del Estado en el cuidado de la infancia" indica que los programas de Acogimiento Familiar se iniciaron en la segunda mitad del siglo $\mathrm{XX}$, sin dar ningún tipo de detalle al respecto. Algunos datos más precisos son aportados en un informe titulado "Situación de niños, niñas y adolescentes sin cuidados parentales en la
República Argentina" elaborado conjuntamente por la Secretaria Nacional de Niñez, Adolescencia y Familia (SENAF) y Fondo de las Naciones Unidas para la Infancia (UNICEF) (Sardá, 2012), así como el libro de Fabiana Isa y María Guasti (2009), quienes aportan su experiencia de trabajo en el programa de Acogimiento Familiar perteneciente a la SENAF.

Aun cuando, nombremos estos casos, es posible señalar dos limitaciones. En primer lugar, la mención a los datos históricos es, solamente, accesoria. En segundo término, refieren como antecedentes de cuidados familiares, tanto a los programas de Amas Externas (que, como veremos, se los ha considerado la primera versión de los programas de Acogimiento Familiar) como a los de Pequeños Hogares. En contrapartida, nosotros, consideramos que son dos programas claramente diferenciados pues los Pequeños Hogares implican, por lo general, una organización interna de tipo institucional mientras que los programas de Acogimiento Familiar refieren a la incorporación de un niño a una vida familiar preexistente. Aquí, nos referimos específicamente a la historia del programa de AF en la provincia de La Pampa.

En lo que respecta específicamente en la provincia de La Pampa, no existe ningún antecedente de investigación científica del programa. En consecuencia, resulta evidente la carencia de antecedentes en general sobre la temática de $\mathrm{AF} \mathrm{y}$, en particular, respecto a la construcción histórica de su institucionalidad, así como a la total ausencia de estudios en nuestra zona geográfica. Esto no resulta novedoso pues, la misma RELAF señala que no existen más trabajos sistematizados que los mencionados y, por su parte, el comité internacional de la ONU sobre niños privados de cuidados parentales, señalaba en el año 2006 la poca información disponible acerca del programa en Argentina (Herrera, 2008; Isa; Guasti, 2009; RELAF 2011; María Sánchez Brizuela, 
coordinadora de contenidos de RELAF, comunicación personal 9 de agosto de 2017).

En la primera parte de este trabajo, realizaremos una reconstrucción histórica del AF en la Provincia de La Pampa. Para ello, a modo de referencia, inicialmente, mencionaremos sus antecedentes en la provincia de Buenos Aires. Luego, describiremos el proceso de constitución del AF en La Pampa. Explicaremos su origen a partir de las políticas públicas provinciales que organizaron en 1967 los "hogares sustitutos", pasando en el año 2000 a llamarse "hogares de contención". Analizaremos los cambios acontecidos en este período, así como las líneas de continuidad plasmadas en la legislación y prácticas instituidas en torno a dichas políticas.

En la segunda parte, nos centraremos en el período posterior al año 2013, cuando la provincia adhirió a la Ley Nacional de Protección Integral de los Derechos de las Niñas, Niños y Adolescentes, que había sido sancionada en el año 2005, para brindar un panorama del estado actual del AF en La Pampa.

Metodológicamente, se trabajó desde un enfoque ex post facto retrospectivo (Montero; León, 2005). Para la primera parte de este trabajo, se utilizaron fuentes secundarias que fueron seleccionadas en función de su referencia a la temática que, es visiblemente acotada. Para la segunda y principal parte de este artículo, se utilizaron fuentes primarias documentales y testimoniales.

En cuanto a las fuentes escritas, se acudió a aquellas a legislaciones, resoluciones y documentación de circulación interna a nivel estatal. Puntualmente, se han utilizado: el decreto 713/67 de la provincia de La Pampa que autoriza al Ministerio de Asuntos Sociales a im- plementar el Programa de Hogares Sustitutos; la resolución 184/67 del Ministerio de Asuntos Sociales de la Provincia de La Pampa que reglamenta los Hogares Sustitutos; un libro que publicó el Ministerio de Bienestar Social con la síntesis de todos los Programas en vigencia, dependientes de la Subsecretaría de promoción y asistencia a la comunidad, en el año 2000; el proyecto elaborado por el equipo profesional de Familias Sustitutas denominado "Reglamentación para el funcionamiento del programa Familias de Contención" del año 2000; la descripción del programa "Familia de Contención" publicado en el sitio Web oficial del gobierno de La Pampa; la Guía de Procedimiento del Sistema de Protección, Promoción y Restitución de Derechos de la Niñez y Adolescencia de la Provincia de La Pampa, elaborada por el Ministerio de Acción Social en el año 2017 y la Ley N. ${ }^{\circ}$ 2703, de adhesión a la Ley nacional N. ${ }^{\circ} 2606$.

Por su parte, las fuentes orales fueron especialmente elegidas por su implicancia con el programa, tanto a nivel de la gestión de estos programas como también, y especialmente, a los equipos técnicos. Una parte de las entrevistas han tenido lugar en el año 2010, en contexto de una investigación anterior, y son las que aportan a la sección histórica. Para la descripción de la situación actual, contamos con los testimonios de los actuales equipos técnicos, cuya participación data del año 2017 y 2018 y han sido entrevistados con un correspondiente consentimiento informado.

\section{Construcción de la institucionalidad en Buenos Aires, Argentina ${ }^{1}$}

En nuestro país, las primeras instituciones benéficas que se ocuparon del albergue de niños, fueron privadas

\footnotetext{
${ }^{1}$ Aun cuando la mayoría de los autores aquí referenciados presentan sus estudios como "nacionales", aquí señalamos que los mismos responden a la reali-
} dad de Buenos Aires, pues no han utilizado fuentes primarias que correspondan a las provincias y no hay referencias a otras latitudes de la nación. 
y siempre a cargo de congregaciones religiosas: la primera Casa de Recogimiento en Buenos Aires se fundó en 1692 y en 1755 se autorizó a la Hermandad de la Santa Caridad a abrir una nueva Casa de Recogimiento para huérfanas (Bising, 2010, 2012).

En el año 1776 se creó el Virreinato del Río de la Plata y Buenos Aires se convirtió en la capital del mismo. Con la llegada del Virrey Vértiz se iniciaron una serie de reformas urbanas de orden y limpieza y, se hizo así evidente una realidad no tan clara hasta entonces: la aparición de cuerpos de niños muertos en las calles. Así, el virrey Vértiz fundó la primera Casa de Niños Expósitos, en 1779, donde la recepción de niños se daba cuando éstos eran hallados en sitios públicos o bien, por medio del sistema del torno (Bising, 2010, 2012; Gómez, 2004). Por las dificultades de mantenimiento la Casa de Expósitos fue entregada a la Hermandad de la Santa Caridad y, para 1815 el reglamento de la Casa confirmaba la pérdida de la Patria Potestad (Bising, 2012).

En 1823 se creó la Sociedad de Beneficenciaorganizada por el ministro Rivadavia- y se hicieron cargo de todas las instituciones de Bien Público destinadas a niños y mujeres regenteadas, hasta entonces, por Órdenes y Hermandades. Obviamente, la casa de Niños Expósitos estaba incluida (Croce, 2007).

Además de estas instituciones de albergue que iban surgiendo, durante el siglo XVII y XVIII la Corona tomó otras medidas a favor de estos "niños desamparados": en 1642, el primer defensor de menores es nombrado por el Cabildo, a petición del Procurador General (Bising, 2010; Gómez, 2004).

En 1814 se creó el Ministerio Pupilar, el Defensor General de Menores debía intervenir en toda causa donde hubiese menores velando por la defensa de sus derechos y seguridad de sus intereses, tanto de los niños huérfanos, pobres, como de aquellos cuyos padres fue- ran declarados incapaces (Bising, 2010). Unos años después, en 1821, se crea el cargo letrado de Defensor de Pobres y Menores quien se ocupaba de los bienes de los varones que eran colocados en un hogar en el que se les habría de enseñar un oficio, a fin de contener así, desde el principio "la mala educación que tantos males depara a la sociedad" (Larrandart, 1991, citado por Bising 2010, pp. 5). Las niñas que no eran ubicadas en casas particulares pasaban al Colegio de Niñas Huérfanas. Unas y otros, difícilmente se librarían del destino de ser criadas y aprendices

En 1871 se crean Asilos de huérfanos para diferentes edades y para uno u otro sexo, en su mayoría dependientes de la Sociedad de Beneficencia. Los infantes sobrevivientes de la Casa de Niños Expósitos, cuando alcanzaban cierta edad, se incorporaban a alguno de ellos (Bising, 2012).

Otra de las prácticas comunes de aquellos años fue la entrega de los hijos de manera voluntaria de los padres a alguna familia de buen pasar económico en donde, a cambio de mantención y educación, el niño prestaba servicios gratuitos a la familia. Por educación se entendían actividades domésticas para las niñas y aprendizaje de oficios para los varones. Esta costumbre se llevaba a cabo tanto por dentro como por fuera del orden jurídico del momento. El Reglamento para las Defensorías de Menores de 1884, permitía a los encargados de las mismas celebrar contratos de locación de los menores con particulares de manera tal que las Defensorías se fueron transformando en agencias de colocación de pequeños sirvientes en casas de familia de modo tal que esta práctica ayudó a opacar el fenómeno del abandono y permitía pasar por alto la prohibición de someter a servidumbre a personas blancas o de color que fueran libres. Esta costumbre, denominada conchabo, estuvo bien arraigada desde el siglo XVIII hasta la 
sanción del Código Civil Argentino, en 1871 (Bising, 2009, 2010, 2012).

Por supuesto, aun cuando aquella vieja costumbre no respondía al formato con el que hoy lo conocemos, la referencia histórica muestra una larga tradición que ha ido cambiando de estructura a lo largo del tiempo. Respecto a las características actuales del programa, sus antecedentes directos son hallados en la década de 1970 cuando surgieron, en algunas provincias, programas de "amas externas" dirigido para albergar niños muy pequeños y luego se fueron modificando, para alcanzar a todas las edades. Los antecedentes encontrados, señalan que, las provincias de Córdoba y La Rioja, fueron las primeras en implementar, a nivel estatal, experiencias de un régimen de AF en el año 1967 y 1968, respectivamente y que, a nivel nacional, el primer antecedente, es la creación del Programa Amas Externas en 1969 (Isa; Guasi, 2009; Sardá, 2012).

Así, cada provincia ha ido implementando estos programas, dependientes del Poder Ejecutivo con diferentes denominaciones, que, por supuesto, también han variado durante estos cuarenta años de aquellos primeros antecedentes: familias solidarias, familias comunitarias, familias sustitutas, familias acogedoras, familias cuidadoras, familias de corazón, familias de abrigo, colocación familiar, etc. Y se le han otorgado diferentes funciones, responsabilidades, aunque todas con el denominador común de dar abrigo a aquellos niños que, por su situación de vulnerabilidad, necesitaban de una contención hasta tanto se tomara una resolución definitiva (Farias-Carracedo; Juárez, 2017; Herrera, 2008).

Un dato fundamental respecto a este tipo de institucionalización es que no se encuentra regulado por una legislación nacional de fondo sino que cada provincia en virtud de su capacidad para normar en materia de políticas públicas tienen la potestad para crear estos "programas" (Herrera, 2008).
A nivel legislativo, sólo la Ciudad Autónoma de Buenos Aires y dos provincias cuentan con una legislación específica sobre Acogimiento Familiar. En la CABA, la ley 2213 del año 2006; la provincia de San Luis con la ley $\mathrm{N}^{\circ}$ IV-0093 del 2004, derogada por una nueva ley en 2013, Ley Nº IV-0871y Tierra del Fuego con la ley 1037 del año 2015.

\section{La construcción de la institucionalidad en la Provincia de La Pampa}

La historia de las instituciones de menores que han albergado niños en situaciones sociales problemáticas en la provincia de La Pampa comienza, aproximadamente, en 1960 con las llamadas macro-instituciones. En esa provincia, existieron cuatro: la guardería "Enriqueta Schmidt" ubicada en Santa Rosa, la capital provincial con una capacidad para 26 niños de ambos sexos, desde los 45 días hasta los 5 años; el Hogar de Niñas “Amelia S. de Bonnet" ubicado, también, en Santa Rosa con una capacidad para 80 niñas de 6 a 18 años; el Hogar de Varones “Dr. José M. Jaramillo” ubicado en la localidad de Eduardo Castex con capacidad para 60 niños de 6 a 12 años y el Hogar Escuela Granja Taller ubicado en General Pico con capacidad para 40 niños varones de 13 a 18 años.

A partir del trabajo de revisión del Ministerio de Bienestar Social acerca del modo de actuación frente a los niños sin cuidados parentales, se fundamentó que las grandes instituciones no eran un lugar adecuado para la formación integral del menor y su socialización y por ello, se propuso un nuevo tipo de institución: los Pequeños Hogares. Este Programa se reglamentó en 1977 y, en 1980, comenzó su efectivo funcionamiento.

Paralelamente a la existencia de las macroinstituciones, en 1967 se reglamentó el Programa de Acogimiento Familiar. Al momento de la reglamenta- 
ción, se denominó "Hogares Sustitutos", nombre que conservó hasta el año 2000 cuando deviene en "Familia de Contención", que sigue vigente hasta la actualidad.

\section{Hogares Sustitutos}

El programa de "Hogares Sustitutos” comenzó en la provincia de La Pampa, en 1967 a partir del Decreto 713 que autorizó al Ministerio de Asuntos Sociales a dictar la Reglamentación así como también a proceder al registro y selección de futuros "Hogares Sustitutos" (Provincia de La Pampa, 1967; Provincia de La Pampa, Ministerio de Bienestar Social, 2000; ex ministra de Bienestar Social de la provincia de La Pampa, comunicación personal).

Cuando se inicia el programa, en 1967, dependía jerárquicamente del Ministerio de Asuntos Sociales, con participación de la Dirección de Bienestar Social y la Dirección General de Protección al Menor (Provincia de La Pampa, 1967). A partir de 1972, debido a las reestructuraciones ministeriales de la provincia pasa a depender del Ministerio de Bienestar Social, Subsecretaria de Promoción y Asistencia a la Comunidad, Dirección de la Familia y el Menor (Provincia de La Pampa, 1972).

A partir del mentado Decreto 713, se reglamentan los Hogares Sustitutos por medio de la Resolución 184/67. La Reglamentación establecía que la finalidad esencial del programa era:

Brindar albergue y cuidados a los niños que por diversas circunstancias deban ser separados del núcleo familiar, en forma temporaria o semi-permanente, proporcionándoles experiencias y educación que en condiciones normales debieran haber recibido en su propio hogar. Ello implica formar íntegramente al niño puesto en guarda, velando por su desenvolvimiento físico y espiritual, desarrollando los valores culturales, estéticos, morales y sociales aceptados por la comunidad (Provincia de La Pampa, 1967, fs.2).

La finalidad primordial de este programa era evitar la internación del menor en instituciones masivas u otro tipo de institutos que no podían brindar una atención personalizada a cada niño, considerando que la promoción de este Programa era la "solución más humana y moderna en la asistencia a niños privados de cuidados dentro de su propia familia" (Provincia de La Pampa, 1967, fs. 3; Provincia de La Pampa, Ministerio de Bienestar Social, 2000). En términos de dos ex asistentes sociales "era una alternativa" para aquellos niños que necesitaban ser separados de sus padres biológicos (asistente social jubilada del Ministerio de Bienestar Social, Delegación Zona Norte, comunicación personal, 30 de junio de 2010; asistente social jubilada, ex directora de la Escuela Granja Taller, ex delegada zonal del Ministerio de Bienestar Social, delegación Zona Norte, comunicación personal, 30 de agosto de 2010)

Como lo señalan algunos de los informantes claves, en la práctica, estas situaciones de acogimiento surgían espontáneamente pues cuando algún niño quedaba sin cuidados parentales, alguien de la familia extensa, amigos, padrinos o vecinos de la familia se hacían cargo de su cuidado. Así, explican que, en sus comienzos, el programa se reglamentó con el objetivo de dar un marco legal a esta situación real así como también aportar con una ayuda económica a los adultos que se hicieran cargo de la crianza de aquel pequeño (asistente social jubilada del Ministerio de Bienestar Social, oficina central de Santa Rosa, comunicación personal, 16 de febrero de 2011; asesor de menores, comunicación personal, 2 de agosto de 2017). De la misma manera, en las Disposiciones Generales del programa se proclamaba que "el régimen de hogares sustitutos es una obra de la comunidad; la función del Estado es ordenadora, de supervi- 
sión de los hogares que se incorporen a este régimen" (Provincia de La Pampa, Ministerio de Asuntos Sociales, 1967, fs. 2).

En lo que refiere a la ubicación, el Programa fue implementado en toda la provincia de La Pampa pues, a través de los años, fueron surgiendo familias guardadoras en distintas localidades (Provincia de La Pampa, Ministerio de Bienestar Social, 2000).

Como ya fue explicado, este Programa consistía en que un matrimonio de la comunidad recibiera en su propio hogar, a un niño. Por ende, los niños se incorporaban al régimen de vida de la familia como si fueran un hijo propio. La familia sustituta recibía un subsidio del Estado en carácter de ayuda familiar por cada menor que se le otorgaba en guarda (Provincia de La Pampa, 1967; asistente social jubilada, ex directora de la Escuela Granja Taller, ex delegada zonal del Ministerio de Bienestar Social, delegación Zona Norte, comunicación personal, 30 de agosto de 2010). La reglamentación establecía que la Familia Guardadora debía poseer una vivienda digna, con comodidad adecuada al número de menores que se le confiaran, aunque no figura un número máximo, sino que todo indicaría que en cada caso particular se evaluaba cuantos niños eran destinados en función de su disposición y de las consideraciones que hiciera el equipo técnico (Provincia de La Pampa, 1967).

Según el reglamento de los Hogares Sustitutos, éstos albergaban a niños desde tres meses hasta 14 años, de ambos sexos (Provincia de La Pampa, 1967). Cuando era posible, se procuraba que los hermanos consanguíneos fueran derivados a un mismo hogar para que permanecieran juntos (asistente social jubilada del Ministerio de Bienestar Social, Delegación Zona Norte, comunicación personal, 30 de junio de 2010).

También se estableció que la familia sustituta tenía la responsabilidad de velar para que entre padres e hijos reinaran relaciones propicias a una vida sana familiar y al desarrollo normal del niño, como así también, a la satisfacción de sus necesidades de orden físico, educativo, afectivo, espiritual y social, en cada etapa de su crecimiento, en el corto o largo plazo que les fuera asignado en guarda (Provincia de La Pampa, 1967).

La reglamentación consideraba que, para constituirse en un Hogar Sustituto, la familia que se postulaba tenía que reunir las condiciones indispensables, la capacidad, "formación moral e ideológica no contraria a los principios de la doctrina occidental y cristiana" y mínima solvencia económica que aseguraran la satisfacción total de las necesidades específicas que estaban destinadas a atender. También se establecía las obligaciones de la Familia Sustituta, así como las causales de suspensión o extinción del derecho a tener niños en guarda (Provincia de La Pampa, 1967, fs. 4).

A pesar de todo lo detalladamente estipulado respecto a la selección y funciones de las Familias Sustitutas, estas no estuvieron exentas de dificultades sino que los testimoniantes señalan que era sumamente difícil encontrar familias que se postularan por vocación, es decir que no se trataba de "familias sustitutas ideales", en palabras de una testimoniante (asistente social jubilada del Ministerio de Bienestar Social, Delegación Zona Norte, comunicación personal, 30 de junio de 2010; Farias-Carracedo; Piñeda, en elaboración).

\section{Familias de contención}

Podemos considerar que, en el año 2000, se inicia una segunda etapa del programa. Para esta época, el organigrama administrativo en el que estaba inmerso el programa, era similar a la etapa anterior: Ministerio de Bienestar Social, Subsecretaría de Promoción y Asistencia a la Comunidad, Dirección General de la Familia 
(Ministerio de Bienestar Social, 2000). Sin embargo, consideramos que en el año 2000 se puede establecer un punto de corte porque el equipo profesional de Familias Sustitutas elaboró un proyecto denominado "Reglamentación para el funcionamiento del programa Familias de Contención (FC)" con el objetivo de revisar algunos aspectos del funcionamiento del programa en función de las nuevas demandas (Busso et al, 2000).

Dicho documento se fundamentó tomando en consideración que, "junto con el aumento de la población, se fue modificando la configuración del grupo familiar, surgiendo nuevas problemáticas como el acrecentamiento de la desorganización familiar y la victimización de niños y adolescentes" (Busso et al, 2000, p. 3). Frente a esto, se consideró necesario una mayor intervención del Estado a los fines de garantizar la protección integral de los niños

Concretamente, los profesionales llamaron la atención acerca del aumento en la cantidad de niños incluidos en familias sustitutas y, por otro lado, los cambios en la motivación de estas familias sustitutas. Es decir, en los orígenes del programa se apelaba a la actitud solidaria de las familias pero, a través de los años, debido a la realidad socioeconómica coyuntural, el aporte económico brindado por el Estado a las familias sustitutas pasó a ser de fundamental importancia como incentivo de inscripción en el programa (Busso et al, 2000).

Teniendo en cuenta, entonces, la creciente demanda del programa y la reconceptualización en relación a la actitud de las familias sustitutas, el equipo técnico consideró fundamental reformular algunos aspectos del funcionamiento del mismo.

La primera modificación se refirió a la denominación, que dejó de llamarse "hogares Sustitutos" para ser "Familia de Contención". Se argumentó a favor de que, "las familias que incluyen un niño en su grupo de convivencia, si bien ocupan un rol esencial en su crianza y formación, de ninguna manera sustituyen las figuras parentales naturales" sino que cumplen un rol complementario al de la familia natural, "que estaría presente en mayor o en menor medida en función de las posibilidades o limitaciones del grupo familiar de origen del niño". Esta nueva perspectiva tenía el objetivo de otorgarles un rol más protagónico a los padres, ofrecerles recursos para que puedan asumir sus responsabilidades parentales en lugar de destituirlos de las mismas (Busso et al, 2000, p. 4).

Según una de las autoras de este documento, alrededor de 1997 habían empezado a trabajar con el equipo de profesionales en miras de modificar aquella reglamentación del año 1967. Como fruto de aquello, elaboraron este nuevo proyecto y que, a pesar de haberse implementado con total legitimación, nunca fue reglamentado oficialmente por la provincia (asistente social jubilada del Ministerio de Bienestar Social, oficina central de Santa Rosa, comunicación personal, 16 de febrero de 2011).

El objetivo fundamental, también se reformula:

El programa "Familias de contención" busca brindar una atención personalizada a niños y adolescentes que por distintas razones, relacionadas a problemáticas sociales de abandono, abuso, violencia, negligencia o incapacidad de los progenitores, no pueden permanecer en sus familias naturales.

Esta atención se realiza a través de la inclusión del niño o adolescente en un grupo familiar que favorezca su desarrollo físico, psíquico y sociocultural, sin que ello implique la desvinculación de su familia de origen (Busso et al, 2000, p. $5)$.

Por su parte, también en este documento se especifican algunos requisitos para los aspirantes a constituirse como FC: podrían ser personas solas, pero, preferentemente casadas; tener entre 30 y 60 años de edad; instrucción primaria como mínimo; ingresos económicos suficientes para sostener a su grupo familiar; acreditar 
buena salud psicofísica; ausencia de antecedentes penales; demostrar capacidad para construir vínculos afectivos con los niños, entre otros.

De la misma manera, se fijaron pautas más precisas para la vivienda tales como: la FC debía ser propietaria de la vivienda que ocupara o, en los casos de alquiler o préstamo, el grupo familiar debía demostrar estabilidad en el lugar de residencia; el mobiliario de la vivienda debía ser adecuado a su finalidad y de acuerdo a las necesidades del grupo de niños que se integraba al hogar; el lugar dentro de la vivienda destinado al niño debía estar integrado al resto de la infraestructura que habitara el grupo familiar (Busso et al, 2000).

Así mismo, se puntean una serie de responsabilidades por parte de la FC para con los niños, la familia biológica o futura familia adoptiva, los profesionales del equipo del equipo técnico y con la Dirección General de la Familia. En el mismo sentido, figuraban las causales de la disolución de una FC como tal (Busso et al, 2000).

El proyecto estableció que la edad de los niños albergados iría entre los 0 y los 14 años, con excepción de los grupos de hermanos. En tales casos, el equipo profesional consideraría especialmente la situación de los adolescentes que superaran la edad indicada para ingresar al programa. La cantidad de niños que podían ser albergados en cada Hogar era hasta tres niños. En aquellos casos de un grupo numeroso de hermanos, se estipulaba que la contención requeriría un marco que excedía las posibilidades de este programa. También en esta etapa, el Estado otorgaba una suma mensual de dinero a la familia de contención por cada niño albergado, en concepto de ayuda para la mantención del mismo (Busso et al, 2000).

Como mencionamos al principio, la reformulación del programa pretendía, entre otros, otorgarle un rol más protagónico a la familia de origen. No obstante, los testimoniantes coinciden con la reglamentación en el sentido de que hubiera sido ideal trabajar con la familia para que, una vez que cambiaran las circunstancias, el niño pudiera reintegrarse, pero aclaran que esto sólo ocurría excepcionalmente (ex ministra del Ministerio de Bienestar Social desde el año 1999 hasta el año 2003, comunicación personal, 20 de agosto de 2010).

Ahora bien, lo anterior tampoco implica que, con esta reformulación, los niños hayan mejorado sus estadías con las FC pues los testimoniantes hacen hincapié en el hecho de que si bien el programa ha sido la mejor alternativa, en relación a otras instituciones de albergue, no estuvieron exentas de gravísimas dificultades referidas desde deficiencias en el manejo administrativo, hasta el maltrato por parte de la familia acogedora (ex psicóloga del Ministerio de Bienestar Social, Delegación Zona Norte, comunicación personal, 19 de julio de 2010; ex psicóloga del Ministerio de Bienestar Social, Delegación Zona Norte, comunicación personal 20 de julio de 2010; asistente social del Ministerio de Bienestar Social, Delegación Zona Norte. Sector administrativo y Programa "Madres", comunicación personal 20 de julio de 2010; ex ministra del Ministerio de Bienestar Social desde el año 1999 hasta el año 2003, comunicación personal, 20 de agosto de 2010.; FariasCarracedo; Piñeda, en elaboración).

\section{El programa en la actualidad}

La provincia de La Pampa fue una de las últimas en adecuar su legislación provincial a la doctrina de la protección integral. El 9 de enero de 2013, la provincia dicta su ley $\mathrm{N}^{\circ} 2703$ en la cual adhiere en los artículos 1 al 42 de la ley nacional 26.061 y a los artículos pertinentes de su decreto reglamentario 415/06, con las disposiciones legislativas provinciales correspondientes 
(Provincia de La Pampa, 2013). El primero de noviembre de dicho año, a través del Decreto N853 se aprueba la Reglamentación de los artículos №40 al 42 y el 30 de diciembre de 2013, por Decreto $\mathrm{N}^{\circ} 1296$ se aprueba la Reglamentación del resto de los artículos de la Ley Provincial. Desde entonces, se ha podido visualizar algunas transformaciones en el programa, en un intento de adecuarse a la ley, que procuramos dejar plasmados aquí.

En el año 2015, como consecuencia del cambio de gestión a nivel del Poder Ejecutivo provincial, se produce una reorganización en el organigrama del mismo $\mathrm{y}$, entro otras modificaciones, el Ministerio de Bienestar Social pasa a denominarse Ministerio de Desarrollo Social (Decreto N¹9/15). El programa de Acogimiento Familiar, que aún conserva el nombre de Familias de Contención, depende de este ministerio (Provincia de La Pampa, Ministerio de Desarrollo Social, Subsecretaría de niñez, adolescencia y familia, Dirección general de niñez, adolescencia y familia, 2017).

La ley de infancia del año 2013 estableció que debía producirse una descentralización de servicios a través de la constitución de espacios locales y/o regionales de promoción y protección de derechos. Las Unidades Regionales (Segundo Nivel de Intervención) y Locales (Primer Nivel de Intervención) fueron definidas como "unidades técnico operativas desconcentradas que tendrán como principal cometido, la atención directa de las situaciones de vulneración de derechos" (Ley N².703 Art. 15).

El objetivo de estas Unidades es la ejecución de programas, planes y servicios de promoción y protección de derechos de niñas, niños y adolescentes (NNyA) pero, cuando la aplicación de las Políticas Públicas no contempla u omite situaciones de amenaza o vulneración de derechos, se requiere políticas de intervención social específicas para hacer cesar o restablecer los derechos amenazados o vulnerados. Estas estrategias se denominan Medidas de Protección Integral de Derechos. Las medidas de protección son múltiples, dependiendo del derecho vulnerado del niño, pudiendo tratarse de restituir su derecho a la educación, a la asistencia en salud, apoyo económico a su familia, entre tantas otras (Provincia de La Pampa, Ministerio de Desarrollo Social, Subsecretaría de niñez, adolescencia y familia, Dirección general de niñez, adolescencia y familia, 2017).

Ahora bien, en aquellos casos en los cuales la vulneración de los derechos de los pequeños dentro del seno familiar llega a un nivel de gravedad tal que los equipos técnicos deciden, como medida de protección, retirar a ese niño del ámbito familiar, ahora, dicha medida, es denominada como "medida de protección excepcional" (Provincia de La Pampa, Ministerio de Desarrollo Social, Subsecretaría de niñez, adolescencia y familia, Dirección general de niñez, adolescencia y familia, 2017). Quien dispone de estas medidas es la Autoridad de Aplicación, que, en esta provincia, está delegada en la Dirección General de Niñez, Adolescencia y Familia (Decreto $\mathrm{N}^{\mathrm{o}}$ 1296/2013; Ley $\mathrm{N}^{\mathrm{o}}$ 2703/2013) quien las define de la siguiente manera:

Las Medidas de Protección Excepcional son medidas de carácter restrictivo ycomo su denominación lo indica - excepcionales, las cuales deben ser dictadas por el menor tiempo posible y sólo ante circunstancias extremas de suma gravedad y urgencia que impliquen la amenaza y/o vulneración que afectan el derecho a la integralidad psicofísica de los niños/ as y adolescentes en el seno de su convivencia familiar. Estos extremos se justifican en tanto una de las particularidades de este tipo de medidas consiste en constituir un remedio de carácter transitorio cuando un niño se encuentra privado o carente de su medio familiar o bien, cuando la satisfacción del interés superior del niño exige que sea separado, es decir que no permanezca en el seno de su 
grupo familiar conviviente (Provincia de La Pampa, Ministerio de Desarrollo Social, Subsecretaría de niñez, adolescencia y familia, Dirección general de niñez, adolescencia y familia, 2017, p. 19).

En este momento, aun cuando en el sitio web oficial del gobierno figuren cinco Unidades Regionales, más la Unidad de la capital provincial (Santa Rosa), nuestros testimoniantes claves han explicado que efectivamente, respecto al programa de Familias de Contención, sólo hay equipos técnicos en tres Unidades con asiento en las localidades de Santa Rosa, General Pico y General Acha. Esta última Unidad Regional, sólo cuenta con un equipo técnico a cargo tanto del área de protección de derechos como de medidas excepcionales. En cambio, para el caso de Santa Rosa y General Pico, los equipos técnicos están diferenciados, es decir, el área de protección de derechos y de medidas excepcionales, tiene su personal respectivamente (Sitio web oficial del Gobierno de La Pampa, 2018; equipo técnico del programa Familias de Contención-ETFC- de la ciudad de General Pico, comunicación personal, 14 de noviembre de 2017 y 26 de marzo de 2018; equipo técnico del programa Familias de Contención-ETFC- de la ciudad de Santa Rosa, comunicación personal, 29 de noviembre de 2017).

Para el cumplimiento de las medidas excepcionales existen dos dispositivos: residencias de adolescentes y familias de contención. Las hoy denominadas residencias de adolescentes corresponden a los viejos Pequeños Hogares que, con algunas transformaciones significativas, siguen cumpliendo aquella función.

Respecto al programa de FC, al día de la fecha, hay tres equipos técnicos en real funcionamiento. Hay un equipo técnico en las ciudades de Santa Rosa y General Pico, que dependen directamente de la Coordinación Provincial de Familias de Contención con su personal exclusivo, y realizan atención en la región centro y nor- te de la provincia, respectivamente. Para el resto de la provincia, está previsto que se ejecute a través de los equipos interdisciplinarios de las Unidades Regionales de Protección de Derechos, aunque, como mencionamos, en este momento solo ejerce tal rol el equipo de General Acha (ETFC de General Pico, comunicación personal, 14 de noviembre de 2017 y 26 de marzo de 2018; ETFC de Santa Rosa, comunicación personal, 29 de noviembre de 2017; Ministerio de Desarrollo Social, Subsecretaría de niñez, adolescencia y familia, Dirección general de niñez, adolescencia y familia, 2017).

A nivel oficial, se proclama que el objetivo del programa es "posibilitar que los niños/as y adolescentes carentes de cuidados parentales, lo hagan de manera excepcional, transitoriamente y por el menor tiempo posible en un núcleo familiar" (Sitio web oficial del Gobierno de La Pampa, 2018). En esta etapa se plasma explícitamente que, una vez tomada esta medida, hay que delimitar alguna de las tres opciones posibles de resolución: restitución al núcleo familiar de origen y/o ampliado; o bien garantizar mediante la figura de la adopción la inclusión a un ambiente familiar estable; o la promoción de un proyecto de Autonomía Progresiva (Provincia de La Pampa, Ministerio de Desarrollo Social, Subsecretaría de niñez, adolescencia y familia, Dirección general de niñez, adolescencia y familia, 2017).

De las tres opciones antes mencionadas, claramente es privilegiada la labor con la familia de origen a fin de procurarle la orientación y condiciones necesarias para abordar las dificultades que ocasionaron la medida dispuesta y facilitar el retorno del pequeño. Ahora bien, si luego de un plazo máximo de 180 días no se hubieran modificado las circunstancias que motivaron la medida, los servicios de protección de derechos deben elevar un informe de conclusión al Juzgado de Familia interviniente, solicitándole el inicio de las acciones civiles que estime corresponder (situación adoptabilidad, restitu- 
ción de guarda, proyecto autónomo) (Provincia de La Pampa, Ministerio de Desarrollo Social, Subsecretaría de niñez, adolescencia y familia, Dirección general de niñez, adolescencia y familia, 2017). Lamentablemente, más allá que se establezca la prioridad de la familia de origen, las profesionales intervinientes en los casos explican que, en la gran mayoría de los casos, la resolución es declararlos en estado de adoptabilidad pues los niños que actualmente llegan a esta instancia están "muy lastimados". En palabras de una de las psicólogas: "llegan en un estado donde el trabajo familiar ya está prácticamente nulo porque han ocurrido tantas vulneraciones, o sea situaciones de abuso y de violencia graves que, a veces, se recurre a la familia de origen para evaluar y fundamentar bien la adoptabilidad[...]. Las presentaciones que están llegando en este momento, es de una gravedad inaudita" (ETFC de Santa Rosa, comunicación personal, 29 de noviembre de 2017).

En cuanto a las dificultades que enfrenta el programa, la principal es que no hay familias de la comunidad que se estén ofreciendo para ejercer este rol lo cual, claramente, atenta con la continuación de este sistema. Frente a esta preocupación, los equipos técnicos diagramaron una fuerte campaña de difusión del programa para captar familias aunque la misma no arrojó frutos (ETFC de General Pico, comunicación personal, 14 de noviembre de 2017 y 26 de marzo de 2018; ETFC de Santa Rosa, comunicación personal, 29 de noviembre de 2017).

Por lo anteriormente mencionado, en este momento, se están dando situaciones inusuales, es decir, no previstas por la reglamentación vigente, que han sido resueltas de maneras alternativas. Entre ellas, la aparición de lo que las profesionales han denominado "referentes afectivos", que son familias de la comunidad que, cuando se han enterado de que una FC ya no desea seguir albergando a un niño en particular con el que ellos han establecido un vínculo, se presentan en el Ministerio para solicitar su guarda. En los hechos, estas familias devienen en FC, pero no han pasado por el proceso de evaluación que como tal les correspondería. Por otro lado, algunos equipos técnicos del área de protección de derechos, toman la medida excepcional "de hecho", entregando al niño a alguien de la familia extensa o a alguien supuestamente allegado al pequeño, sin derivar el caso al equipo técnico de FC. A lo anterior, se suma el albergue de niños en hoteles pues, ante la urgente necesidad de retirarlos de sus hogares y la ausencia de familias dispuestas a recibirlos transitoriamente ni la existencia de residencias para ellos, no han encontrado otra solución (ETFC de General Pico, comunicación personal, 14 de noviembre de 2017 y 26 de marzo de 2018; ETFC de Santa Rosa, comunicación personal, 29 de noviembre de 2017).

También en esta etapa, el programa está diseñado para NNyA menores de 18 años (Sitio web oficial del Gobierno de La Pampa, 2018). En cuanto a la cantidad de niños, si bien mes a mes hay ingresos y egresos, hemos seleccionado el mes de marzo como punto comparativo. Para marzo de 2015, había 168 niños: en el año 2016, 152; en el año 2017, 65. Por último, en marzo de 2018, había 47(datos aportados por el equipo técnico de gestión, del Ministerio de Acción Social, Provincia de La Pampa).

Como puede verse en los datos anteriores, ha habido una disminución significativa de los niños integrados a este programa. Posiblemente, el motivo sea multicausal. Por un lado, los equipos técnicos refieren que había muchos "casos viejos", es decir, situaciones de niños que habían ingresado en el programa bajo la vieja ley y hacía muchos años que estaban conviviendo con una familia de contención. En estos casos, se procuró darles alguna salida definitiva (restitución a la familia biológica, guardas judiciales a las familias de conten- 
ción) y, de este modo, darles de baja en el programa. Por otro lado, han aumentado las ayudas económicas a las familias biológicas sumado a la implementación de la figura del acompañante familiar, que es una persona que, desde el Ministerio, se pone a disposición de la familia de origen para apoyar, a la madre biológica, en el desempeño de su rol. Por último, esto está en clara correspondencia con aquellas situaciones de albergue que hemos llamado inusuales que, por supuesto, logran que no figuren entre las listas de niños acogidos en el programa en estudio (ETFC de General Pico, comunicación personal, 14 de noviembre de 2017 y 26 de marzo de 2018; ETFC de Santa Rosa, comunicación personal, 29 de noviembre de 2017).

En cuanto a la zona geográfica de implementación de esta modalidad, si bien se entiende que-en las tres etapas que hemos señalado- estuvo diseñado para toda la provincia, aquí consideramos importante apuntar esto debido a que el informe de la Red Federal señala que “existe la práctica del acogimiento en las localidades de Santa Rosa, General Acha y General Pico" (Red Federal de Acogimiento Familiar, 2018, p. 43). Seguramente, la confusión responde a los lugares donde hay equipos técnicos específicos. En este sentido, valiéndonos de las mismas planillas de conteo del mes de marzo desde el 2015 hasta el 2018, se han registrado las siguientes localidades pampeanas con FC: Winefreda, Catriló, Bernardo Larroudé, La Maruja, 25 de mayo, Toay, Anguil, Macachin, Luan Toro, Colonia Barón, Lonquimay, Macachin, Vertiz, Uriburu y, por supuesto, en Santa Rosa, General Pico y General Hacha (datos aportados por el equipo técnico de gestión, del Ministerio de Acción Social, Provincia de La Pampa).
Hemos presentado aquí el recorrido histórico del programa de Acogimiento Familiar en la provincia de La Pampa, Argentina, desde sus orígenes, en 1967 hasta la actualidad. En esta linealidad, hemos establecido una periodización en tres etapas. La primera etapa, que va desde el inicio en 1967 hasta el año 2000. La segunda, desde el año 2000 hasta el 2013 y, por último, desde el año 2013 hasta nuestros días. El principal justificativo del punto de corte entre la primera y segunda es, el cambio de denominación y la aparición de un nuevo documento oficial que estableció diferentes pautas de funcionamiento. La última etapa, claramente encuentra su principal argumento en la adhesión de la provincia a la ley de protección integral, lo cual implica-desde lo declarativo-un cambio esencial en doctrinas de infancias.

Respecto a la primera etapa, queremos señalar el lugar pionero que ha tenido La Pampa en el asunto pues, cuando los antecedentes históricos indican que las provincias de Córdoba y La Rioja, fueron las primeras en implementar, a nivel estatal, experiencias de un régimen de acogimiento familiar en el año 1967 y 1968, respectivamente y que, a nivel nacional, el primer antecedente, es la creación del Programa Amas Externas en 1969 (Isa; Guasi, 2009; Sardá, 2012), observamos que nuestra provincia en estudio también ha estado a la altura de aquellas experiencias que la escasa bibliografía menciona. Dicho esto, también consideramos la posibilidad de que no se trate de situaciones aisladas de tres provincias sino que hayan existido en la realidad pero, lo que no hay, son estudios históricos que pongan en valor lo acontecido en materia de infancia en regiones geográficas “periféricas”. En esta dirección, alentamos a nuestros colegas a embarcarse en este tipo de estudios, sumamente necesarios para la construcción del conocimiento en un sentido verdaderamente federal y latinoamericano.

\section{Discusiones y conclusiones}


Desde otro lugar, queremos invitar a todos aquellos interesados en la temática a la lectura detallada de aquella primera ley de Hogares Sustitutos del año 1967, pues sus postulados no distan demasiado de las ultimas legislaciones y ya, en aquel entonces, se planteaba como la "solución más humana y moderna" (Provincia de La Pampa, 1967, fs. 3), lo cual debería inducirnos a una verdadera reflexión y cuestionamiento de las practicas que van construyendo las instituciones.

En cuanto a la segunda etapa, fundamentalmente queremos resaltar la intención de los equipos técnicos por otorgarles a las familias biológicas un rol más protagónico en la responsabilidad para con sus hijos. En este sentido, es posible advertir que cuando hoy se señala que la Ley de Protección Integral privilegia a la familia de origen del niño e indica que las medidas excepcionales se deben implementar bajo formas de intervención no sustitutivas del grupo familiar de origen (Ley $\mathrm{N}^{\circ} 26061$, Art. 41), no son planteos novedosos, al menos, para esta provincia.
Por último, en lo que respecta a los últimos años del funcionamiento del programa, sin desconocer el esfuerzo que la provincia viene realizando por adecuar sus prácticas a la legislación, no podemos dejar de reflexionar acerca de estas situaciones inusuales que, posiblemente, sean el reflejo de la aparición de nuevas problemáticas a nivel social y que, como tales, requerirán de nuevas estrategias y replanteos del funcionamiento de este programa. Es, justamente, en este sentido que deseamos de manera urgente poder aunar esfuerzos desde los diversos actores interesados en la temática, para repensar y reelaborar medidas. Consideramos fundamental no sólo intentar adecuar las prácticas a la legislación sino, cuestionar las mismas prácticas pues son ellas las que van construyendo la institucionalidad del programa. Tal vez, en ese cuestionamiento sumado al conocimiento de otras prácticas en otras latitudes geográficas, en otros contextos, nos permitan construir nuevas institucionalidades más adecuadas en la protección de los derechos de nuestros niños. 


\section{Referencias}

BISING, Nidia Elinor. Prácticas y debates socio-jurídicos en torno al lugar de los niños en el espacio familiar y social - Postrimerías del siglo XIX. Opinión Jurídica, 15(8), 2009, p. 151 -165.

BISING, Nidia Elinor. La infancia en debate. Hijos ilegítimos y abandonados delincuentes en Córdoba (18711914). Estudios Digital, III, 2010, s/p.

BISING, Nidia Elinor. La Relación Estado Familia e Infancia en la Argentina. El Proceso de Construcción de la Infancia. En, Bising, Nidia Elinor, La construcción socio jurídica de la infancia-Córdoba, Argentina. Siglos XIX y XX. Berlín: Editorial Académica Española, 2012.

BUSSO, Adriana; TORTA, Marcela; MONTIGEL, María; CAMILATTI, Federico: Proyecto de Reglamentación para el funcionamiento del Programa Familias de Contención. Departamento de Promoción Familiar. Dirección general de la Familia. Ministerio de Bienestar Social. Provincia de La Pampa. Mimeo, 2010. Gentileza de un testimoniante.

CROCE, Pablo. La Casa Cuna De Buenos Aires. Epopeya En Cuatro Siglos. Buenos Aires: Fundación Hospital Pedro de Elizalde, 2017.

FARIAS-CARRACEDO, Carolina. \& JÚAREZ, Ana Rocío. ¿Qué les pasa a estos niños? Respuestas desde los saberes psi en Buenos Aires (1900-1940). Revista Psicología e Saúde. En prensa.

FARIAS-CARRACEDO, Carolina; PIÑEDA, María Andrea. "No se puede adoptar": análisis histórico de objetos discursivos que determinan decisiones judiciales sobre niñas y niños en la provincia de La Pampa. XVIII Encuentro Argentino de Historia de la Psiquiatría, Psicología y Psicoanálisis. 12, 13 y 14 de octubre de 2017. Rosario, Argentina.

FARIAS-CARRACEDO, Carolina; PIÑEDA, María Andrea. ¿Acogimiento Familiar o familias adicionadas? análisis histórico de las dificultades con los padres de acogida. En elaboración.

FERNÁNDEZ-HASAN, Alma. Familias Cuidadoras: construcción familiar social. Revista Argentina de Humanidades y Ciencias Sociales, 6 (2), 2008, s/p.

FERNÁNDEZ-HASAN, Alma. Vínculos y subjetividades: las mujeres como madres en el Programa Familias Cuidadoras de la provincia de Mendoza. San Luis, Argentina, tesis (doctorado), Universidad Nacional de San Luis, 2011.

FERNÁNDEZ, Julieta. Funcionamiento familiar y la tendencia a la apropiación en familias Temporarias. Mendoza, Argentina, tesina (Licenciatura), Universidad del Aconcagua, 2010.

GOBIERNO DE LA PAMPA, MINISTERIO DE BIENESTAR SOCIAL, SUBSECRETARÍA DE PROMOCIÓN Y ASISTENCIA A LA COMUNIDAD. Formulación de los programas en ejecución. 2000.

GOBIERNO DE LA PAMPA, MINISTERIO DE DESARROLLO SOCIAL (2018). Programa Familias de Contención. Sitio web oficial. Disponible en $<$ http://mbs.lapampa.gov.ar/programa-familias-de-contencion.html $>$ acceso el $28 / 02 / 2018$.

GOBIERNO DE LA PAMPA, MINISTERIO DE DESARROLLO SOCIAL (2018). Programa Provincial Unidades Regionales de Protección de Derechos de Niños, Niñas y Adolescentes. Disponible en <http:// www.mbs.lapampa.gov.ar/fiscalidad-regionales.html> acceso el 8/03/2018.

GÓMEZ, Daniel. Genealogía del concepto de Patronato de Menores. Prácticas institucionales desde el torno de la Casa de Expósitos a la ley 10.903. En: II Congreso Nacional de Sociología. VI Jornadas Nacionales de Sociología, Buenos Aires, 2004. Actas del II Congreso Nacional de Sociología. VI Jornadas Nacionales de Sociología “ ¿Para qué la sociología en la Argentina actual?". Buenos Aires: Editorial Carrera de Sociología, s/p.

HERRERA, Marisa. Familias Cuidadoras, Familias Solidarias y Acogimiento Familiar en el derecho argentino. V Foro "Derecho de la Infancia y de la Adolescencia". 3, 4, y 5 de diciembre de 2008. Caracas, Venezuela.

ISA, Fabiana; GUASTI, María. Acogimiento Familiar y Adopción. Un aporte interdisciplinario en materia de infancia. Buenos Aires: Espacio, 2009.

LUNA, Matilde. Menores en riesgo y acogimiento familiar. Compartir el compromiso. Buenos Aires: Lumen Hvmanitas, 1994 
LUNA, Matilde. El quehacer con menores y familias. Buenos Aires: Lumen Hvmanitas, 1998.

LUNA, Matilde. Acogimiento familiar: respuesta social y de estado en el cuidado de la infancia. Buenos Aires: Lumen Hvmanitas, 2001

LUNA, Matilde. Vínculos en la infancia. Nuevas contribuciones al acogimiento familiar. Buenos Aires: Lumen Hymanitas, 2005

LUNA, Matilde. Una mirada latinoamericana al Acogimiento Familiar. Buenos Aires: Lumen Hymanitas, 2009.

MONTERO, Ignacio \& LEÓN, Orfelio. Sistema de clasificación del método en los informes de investigación en psicología. Revista Internacional de Psicología Clínica y de la Salud, 5 (1), 2005, p. 115-127.

PROVINCIA DE LA PAMPA, MINISTERIO DE DESARROLLO SOCIAL, SUBSECRETARÍA DE NIÑEZ, ADOLESCENCIA Y FAMILIA, DIRECCIÓN GENERAL DE NIÑEZ, ADOLESCENCIA Y FAMILIA. Guía de Procedimiento del Sistema de Protección, Promoción y Restitución de Derechos de la Niñez y Adolescencia de la Provincia de La Pampa. Documento base del Sistema de Información Integrado de Niñez y Adolescencia (SIINyA). 2017. Mimeo. Gentileza del equipo técnico de gestión de la Dirección General de Niñez, Adolescencia y Familia.

RED LATINOAMERICANA DE ACOGIMIENTO FAMILIAR-RELAF- Sitio Web Oficial (s/f). $<\underline{\text { https:// }}$ WWW.relaf.org/>

RELAF\&UNICEF. Orientaciones para la capacitación. Guía de estándares para el personal de las entidades públicas y privadas que se ocupan de la protección de derechos de niñas, niños y adolescentes. 2010a. Disponible en http://www.relaf.org/guia\%20adultos.pdf, acceso el 10/07/2017.

RELAF\&UNICEF. Las voces de los niñas y niños. 2010b. Disponible en https://www.relaf.org/materiales/ Voces.pdf, acceso el 10/07/2017.

RELAF\&UNICEF. Acogimiento Familiar. Guía de Estándares para la práctica. 2010c. Disponible en $<$ https:// www.relaf.org/materiales/Acogimiento\%20Familiar.pdf $>$ acceso el 22/07/2017.

RELAF: La situación del acogimiento familiar en Argentina. Informe preliminar. 2011. Disponible en <http:// www.relaf.org/informe_af.html $\geq$. Acceso el 02/06/2015

RELAF. Hacia un diagnóstico de la situación de los niños internados en instituciones de cuidado residencial en América Latina y el Caribe. 2013. Disponible en <http://www.relaf.org/Presentaciones2013/Matilde\% 20Iniciativa\%20Regional.pdf $\geq$ acceso el 10/07/2017.

RED FEDERAL DE ACOGIMIENTO FAMILIAR. Creación de condiciones y lanzamiento de la red. 2016. Mimeo. Gentileza de la Red Federal de Acogimiento Familiar

RED FEDERAL DE ACOGIMIENTO FAMILIAR (2018). Primer Informe. Situación actual del acogimiento en Argentina. 2018. Disponible en <https:/www.unicef.org/argentina/spanish/Primer_Informe_RED.pdf $>$ acceso el $15 / 01 / 2018$ de

SARDÁ, Laura. Situación de Niños, Niñas y Adolescentes sin Cuidados Parentales en la República Argentina. Buenos Aires: Ministerio de Desarrollo Social de la Nación - Fondo de las Naciones Unidas para la Infancia (UNICEF), 2012.

VALGAÑÓN, Mónica. Estilo de funcionamiento de las familias de acogida y conducta adaptativa - autoconcepto de los niños, niñas y adolescentes bajo su cuidado. Salud \& Sociedad, 5 (2), 2014, p. 156 - 169.

Submissão: 19/04/2018

Aceite: 02/10/2018 